\title{
Assessing Investigator Site Systems for Originating Source Data for Regulated Clinical Research
}

\section{Suzanne Bishop ${ }^{1 *}$, Cheryl Tucker McClung ${ }^{2}$, Yvonne Rollinger ${ }^{3}$, Yiannis Karageorgos ${ }^{4}$, Kelley Olree ${ }^{5}$, Ludger Bührmann $^{6}$ and Martijn I Griep ${ }^{7}$}

${ }^{1}$ North America Facilitator, eClinical Forum, USA

${ }^{2}$ Design Hub Consultant--Data Sciences, Lilly, USA

${ }^{3}$ Managing Director, OmniComm Europe GmbH, USA

${ }^{4}$ External Data Integration Lead, Bristol-Myers Squibb, USA

${ }^{5}$ Study Manager, Pfizer, USA

${ }^{6}$ Head of Monitoring, GlaxoSmithKline, USA

${ }^{7}$ Associate Director, Johnson and Johnson, USA

*Corresponding Author: Suzanne Bishop, North America Facilitator, eClinical Forum, USA.

Received: April 24, 2019; Published: June 20, 2019

DOI: $10.31080 /$ ASPS.2019.03.0321

An increasing number of healthcare institutions are employing Electronic Health Records (EHRs) and other electronic systems to handle patient data, many of the data points needed for clinical research are originating in EHRs, making the EHRs "eSource" for clinical research. Even if these data points are not used in their electronic state for clinical research, but are printed from the EHRs and then re-entered into an EDC (Electronic Data Capture) system for a clinical trial, the source of the information must still be confirmed as compliant with standards set forth in regulations and applicable guidance documents.

The eClinical Forum, a transatlantic, not-for-profit and noncommercial, technology independent group representing members of the pharmaceutical, biotechnology, and allied industries has produced an assessment form that is available, for free, to determine if a site's system can source data that will be used in a regulated clinical trial.

The eSource Readiness Assessment (eSRA) contains questions based on regulations and regulatory agency guidelines for clinical research data sources from FDA, EMA, PMDA, and ICH as identified in the eClinical Forum Requirements for Electronic Data for Regulated Clinical Trials ${ }^{1}$. In July 2018, the FDA issued a Guidance for Industry: Use of Electronic Health Record Data in Clinical Investigations, which states: "Sponsors and clinical investigators should ensure that policies and processes for the use of EHRs at the clinical investigation site are in place and that there are appropriate security measures employed to protect the confidentiality and integrity of the study data". This Guidance further stresses the need for an assessment such as eSRA and identifies key areas that should be as- sessed, all of which are contained in the eSRA questions. It further states that it is the sponsor's responsibility to ensure that the site environment (both system and process) is appropriate for collecting and managing data used for clinical research.

Any system that is originating patient data that is used in a regulated clinical trial should be evaluated for its suitability, however only areas/modules of an electronic system that are being used to enter, store, manage, or otherwise handle records that will be used for clinical research need to be evaluated. For example, the portion of an EHR system that might be used to handle insurance claims would not need to be evaluated. Following is an example list and not an exhaustive list of systems that might provide data used for regulated clinical research:

- $\quad$ Electronic Health Record Systems (EHRS) or Electronic Medi cal Record Systems (EMRS)

- Laboratory/Diagnostic Systems

- Imaging Systems (e.g. X-ray, CT scan)

- Pharmacy Systems (if used to hold records of patient medica tion dosing)

- Radiology Systems

The eSRA tool is of great benefit to all players in the clinical research realm. In particular, the following should be noted:

Clinical Investigators

- Clinical Investigators are encouraged to use this checklist to assess systems, for which they are responsible, that collect data that may be used in a clinical trial.

1"eCF Requirements for Electronic Data for Regulated Clinical Trials V2018" is available to the public at www.eclinicalforum.org. V2019 is available only to eClinical Forum members. For membership information, please see www.eclinicalforum.org/membership 
- A separate checklist should be used for each system at a site that will be used as source for clinical research patient records.

- A site should be aware that a "no" answer to a question does NOT mean that the site will be rejected, but rather that the sponsor will work with the site to ensure that any potential risk is mitigated. Some questions have an asterisk * next to the Yes (Yes*), indicating this item is "strongly recommended". If a site is not compliant with these questions, we recommend that their computerized system is not used to source clinical research data until this item can be answered "yes".

- Investigator site responsibility with respect to system installation, validation and maintenance may be handled by their organization's IT department and/or a vendor. In these cases, the investigational site is still responsible for ensuring that these other parties are fulfilling these responsibilities for any systems providing data used in clinical research.

- Sites should retain a record of this assessment for use in improving their system and processes and to assist them with future system assessments. Further, sites should have a documented site process regarding how source data are collected and managed.

- An investigator can avoid multiple requests from different sponsors for information pertaining to regulatory appropriateness of their system and processes, as the same completed eSRA Assessment can be given to each sponsor they work with.

\section{Sponsors/CROs}

- Sponsors are encouraged to ask sites to use this assessment tool to determine if a site is ready for sourcing regulated clinical research from their computerized systems.

- Sponsors should obtain a completed assessment from their site and this assessment should be retained in their trial master file.

- Sponsors may have additional questions for determining if a site is suitable for their clinical trial; however those provided by eSRA are the minimum requirements as based on US, EU and Japan regulations, guidance documents, directives and reflection papers. A list of all regulatory documents used as a basis for eSRA can be found at www.eclinicalforum.org/esra. aspx.

- eSRA does not "certify" or "qualify" but merely provides information that the sponsor can use to determine the appropriateness of using data that originated in a healthcare system.
- It is up to each individual sponsor to review a completed site assessment to determine if the site is appropriate for their clinical trials. The eSRA assessment does not provide certification or an "answer" as to whether the site as a whole is appropriate - it only indicates the site responses to each question. Some questions have an asterisk * next to the Yes (Yes*), indicating this item is "strongly recommended". If a site is not compliant with these questions, we recommend that their computerized system is not used to source clinical research data until this item can be answered "yes".

- Sponsors should work with their sites to ensure that any potential risk is mitigated. If after reviewing a completed eSRA from a site, the sponsor believes there are too many risks to warrant using the system to provide source data, then the system (and perhaps the site) should not be used for clinical research. However, the site may be given a period of time to make necessary adjustments to comply with eSource standards based on the completed assessment, and may ultimately comply such that the system can be used for source data for clinical research.

\section{Regulators}

- Regulators benefit by a common assessment that is mapped to US, EU and Japan regulations, guidance documents, directives and reflection papers.

- The eClinical Forum commit's to updating the eSRA questions as would be needed when updates are made to the underlying documents and/or new pertinent documents are released from any of the regulatory authorities listed above. The eSRA Handbook and Assessment template can be downloaded for free at www.eclinicalforum.org/esra

- All are encouraged to provide feedback to the eClinical Forum regarding interpretations of regulations, eSRA questions, etc.

\section{Volume 3 Issue 7 July 2019 \\ (C) All rights are reserved by Suzanne Bishop., et al.}

'The eClinical Forum's mission is to serve the clinical research industry by focusing on those systems, processes and roles relevant to electronic capture, handling, and submission of clinical data. For further information on the eClinical Forum, visit the website at http://www.eclinicalforum.org. 